\title{
Do depth shifts resulting from an interocular delay in stimulation result from a breakdown of binocular fusion? ${ }^{1}$
}

\author{
EUGENE R. WIST ${ }^{2}$ \\ FRANKLIN AND MARSHALL COLLEGE
}

\begin{abstract}
Previous studies have shown that the introduction of an interocular delay in presentation to the two eyes of a briefly viewed, haploscopically presented object resulted in a rearward shift in the depth position of this object. The present study investigated the magnitude of this depth shift as a function of flash duration and determined the relationship of this magnitude to two-flash and simultaneity thresholds. It was found that depth shift magnitude decreased as flash duration increased, and that large depth shifts were associated with lange two-flash thresholds. The hypothesis that depth shifts can occur within the temporal limits of binocular fusion was supported.
\end{abstract}

Two previous studies have reported that the introduction of an interocular delay in presentation to the two eyes of a briefly viewed, haploscopically presented test object results in a rearward shift in the perceived depth position of this object. In the first study (Wist \& Gogel, 1966), it was found that as the interocular delay interval (IDI) was increased from 0 to $96 \mathrm{msec}$, the haploscopically viewed test object appeared increasingly behind a continuously illuminated binocular standard. The second study (Wist, 1968) was concerned with the reason for this rearward depth shift. In the first study, the hypothesis was advanced that there is a temporal continuum between binocular and monocular stimulation. This hypothesis stated that up to a critical delay, binocular fusion is maintained and therefore no shift in the depth position of the test object occurs. Beyond this critical delay, the now essentially monocular test object's position is determined by such variables as Panum's Limiting Case (Ogle, 1962) and the equidistance tendency (Gogel, 1965), which either cannot operate when binocular fusion of the test object occurs (Panum's Case) or operate with less effectiveness (the equidistance tendency).

In this second study, an attempt was made to produce a forward depth shift by opposing Panum's Case (which would predict a rearward shift) with an equidistance tendency that would produce a forward shift. It was found that, while the test object could be made to shift forward in perceived position when it was presented monocularly, no forward shift in depth was obtained when the IDI was increased from 0 to $64 \mathrm{msec}$. Instead, the shift was rearward. Two possibilities for this failure of the equidistance tendency to "override" the Panum effect were discussed. One was that at the longest IDI used-64 msec-the Panum effect was still much stronger than the equidistance tendency. Therefore, in order to obtain a rearward shift in the perceived position of the test object, longer IDIs must be used. This possibility remains to be tested. The other possibility was that depth shifts contrary to the initial hypothesis can occur within the temporal limits of binocular fusion because temporal disparity of stimulation has an effect on perceived depth equivalent to that of retinal (spatial) disparity. This hypothesis implies that the visual system treats small temporal disparities identically to small spatial disparities of stimulation. Thus, according to this hypothesis, depth shifts can occur even though the IDI is not large enough to make the test object effectively (functionally) monocular as would be required by the initial hypothesis tested.

The present study is concerned with this latter possibility. If depth shifts can occur within the temporal limits of binocular fusion, and if the dichoptic two-flash threshold is used as a measure of the temporal limit of binocular fusion (Ireland, 1954), several empirical consequences should result. For example: (1) Any variable that affects the temporal limits of binocular fusion as measured by the dichoptic two-flash threshold ought to affect depth shift magnitude. The smaller the two-flash threshold, the smaller the temporal range within which a depth shift can occur. Thus, smaller depth shifts would be expected when conditions are arranged to produce small two-flash thresholds. (2) The threshold for a depth shift should be smaller than the threshold for the perception of two flashes.

The present study investigates the first consequence. A test of the second will be reported later. Mahneke (1958) reported that the two-flash threshold decreased rapidly as flash duration increased from 1 to $50 \mathrm{msec}$. Accordingly, two flash thresholds were obtained for flash durations of 5,20 , and $40 \mathrm{msec}$ in the present study, and depth shift functions were obtained with IDIs of $0,20,30,40$, 50,60 , and $70 \mathrm{msec}$ for each of these flash durations. On the hypothesis that depth shifts occur within the temporal limits of binocular fusion, it was predicted that larger depth shifts would be obtained for the shorter flash durations, which would produce larger two-flash thresholds.

\section{Subjects}

\section{METHOD}

Eight paid undergraduate male students, each possessing a visual acuity of $20 / 20$ or better, normal lateral and vertical phoria, and a stereoacuity of at least $25 \mathrm{sec}$ of arc were employed as Ss. All the above measurements were made with a Bausch and Lomb Master Orthorater. In addition, each $S$ received a refraction by an optometrist. No astigmatism was indicated.

\section{Apparatus}

A Roush flash generator (Roush \& Hamburger, 1948) was used to drive two Sylvania R1 131C glow modulator tubes to provide appropriate flash durations, interocular delay intervals (IDIs), and repetition intervals. The disk (see Fig. 1A) was generated by coupling two reflex sights so that the image of a disk reticle $30 \mathrm{~min}$ in diam, registered upon each eye through the optical systems of the reflex sights, was projected at optical infinity in the median plane down a visual alley by means of beam splitters. The two glow modulator tubes were used to transilluminate the disk reticles, one for each eye.

The vergence angle between the images of the two disk reticles was adjusted by each $S$, so that the apparent depth position of the binocularly fused disks was the same as that of the standard stimulus physically located $710 \mathrm{~cm}$ away from $S$ down the visual alley (see Fig. 1A).

The standard stimulus was a white, binocular, transilluminated rectangle, $14 \mathrm{~min}$ wide and $50 \mathrm{~min}$ high with a luminance of $1.5 \mathrm{ft}-\mathrm{L}$, located $66 \mathrm{~min}$ to the right of the apparent position of the disk. A binocular point source with an apparent brightness equal to that of the standard stimulus was located $38 \mathrm{~min}$ below the bottom of the disk. By means of an instantaneously reversible motor, the depth position of this point source was 
Fig. 1. Scale diagram of stimulus situation. (A) Angular dimensions and separations of the disc and standard stimulus in angular units (minutes of arc). (B) Stimulus arrangement for brightness matching task. The disk was monocularly projected onto the larger projection surface appearing as a black annulus in this figure. Disk intensity was adjusted by $S$ so that its brightness matched that of the large rectangle.

under S's control. It moved at the rate of $8.5 \mathrm{~cm} / \mathrm{sec}$ and was used to determine the apparent depth position of the binocularly fused disk as a function of IDI and flash duration. The disk for the right eye contained a black vertical bar, as indicated in Fig. 1A. The disk for the left eye, not shown in this figure, contained a horizontal bar of identical appearance. With short IDIs, S saw them simultaneously in the form of a cross. Their relevance to the experiment is discussed below.

\section{Brightness Matching Task}

Since it was desired that flash duration alone be varied, a brightness matching procedure was employed to insure that flashes were equal in brightness across durations. To accomplish this, the disk was monocularly projected onto the surface of a large rectangle $1.5 \mathrm{ft}-\mathrm{L}$ in intensity located in the median plane $930 \mathrm{~cm}$ away from $S$ (see Fig. 1B). This rectangle was present only during brightness matching. A black disk was located in the center of this rectangle so that when the reticle disk was projected onto it, $S$ observed a flashing disk surrounded by a continuously illuminated background. By means of a neutral-density wedge, $S$ was able to adjust the intensity of the flashing disk until it matched this background in brightness. This was done independently for each eye. Each experimental session began with this matching procedure for the flash duration to be employed during the remainder of the session. The repetition interval of the flashing disk was always $1 \mathrm{sec}$, for this task as for all experimental tasks, to insure temporal independence of flash pairs (Wist \& Gogel, 1966).

\section{Depth Shift Task}

The S's task in this phase of the experiment was to position the point source in depth so that it appeared equidistant to the disk (see Fig. 1A). Fixation was maintained on the cross in Fig. 1A for this task as well as for others. IDIs were $0,20,40$, and $60 \mathrm{msec}$ for one-half of the sessions and $0,30,50$, and $70 \mathrm{msec}$ for the remainder. The left eye was always stimulated first. Flash duration did not vary within sessions. Four trials
$A$

STANOARD

STIMULUS
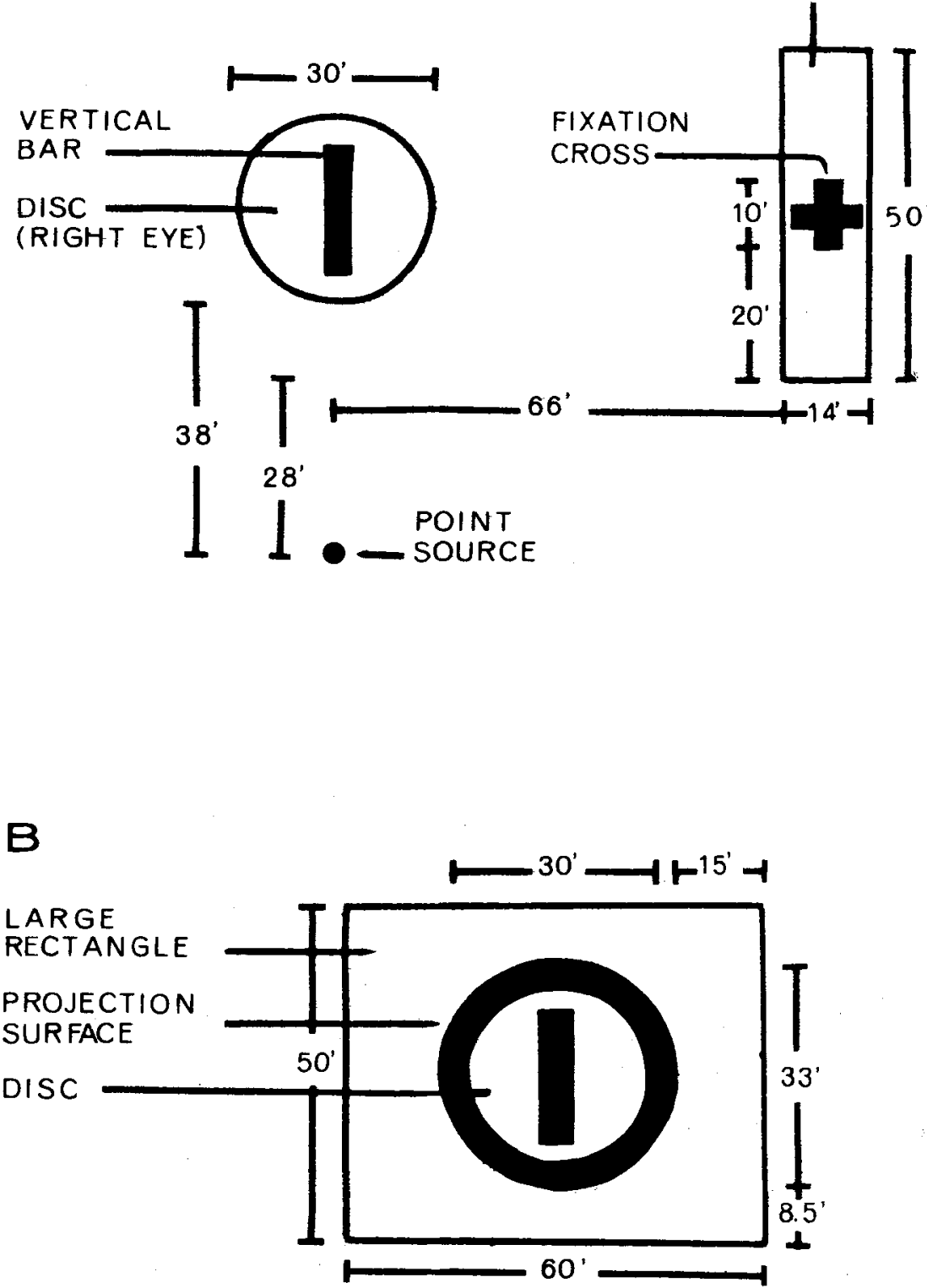

were run for each IDI, for a total of 16 trials per session per $S$. The four initial trials of each session involved a 0 -msec IDI. In addition, four terminal trials with an IDI of $0 \mathrm{msec}$ were given in order to check the stability of the perceived position of the disk when no interocular delay was administered. Nonzero delays were counterbalanced within and across sessions.

During a typical session, $S$ first positioned the disk with an IDI of $0 \mathrm{msec}$ and a repetition interval of $1 \mathrm{sec}$ so that it appeared equidistant to the standard stimulus. Then he positioned the point source so that it appeared equidistant to the disk. For this task, four trials were run, two "ascending," in which the point source was clearly behind the disk at the onset of a trial and moved toward $S$, and two "descending," in which the point source was clearly in front of the disk and moved away from $S$. S was instructed to stop the point source when it appeared equidistant to the disk. He was allowed to "trim" his adjustment. Ascending and descending trials were administered in a counterbalanced order.

After the above four trials, the IDI was increased and the above procedure repeated. A depth shift consisted of the difference between the mean position of the disk at 0 -msec IDI and the mean position of the 
disk at each of the nonzero IDIs. A positive difference always indicated that the disk shifted away from $S$ in perceived position.

\section{Two-Flash Threshold Task}

On each trial during the depth shift sessions, $S$ was required to indicate whether he saw one or two flashes immediately after completing the depth shift task. If he saw one flash, he indicated this by pressing a button that activated a buzzer once. If he saw two flashes, he pressed the button twice.

In addition, nine separate sessions were run after the depth shift sessions in which two-flash thresholds were obtained for each of the three flash durations. These data were obtained using the method of constant stimuli with six additional Ss who met the original screening criteria.

Ireland (1954) found that two-flash thresholds under dichoptic viewing conditions were quite variable and unstable. One possible reason for this is S's difficulty in determining a criterion for "two" flashes. In order to facilitate the formation of a stable criterion, three response categories were made available to $S$ for the first three of the nine sessions. They were "one," "flick," and "two." The instructions defined the "flick" category as a brief dimming or lowering of illumination during the course of a flash.

After the completion of the above three sessions, one at each of the three flash durations, another block of three sessions was run in which $S$ had available only the "one" and "flick" categories. A final block of three sessions was run in which only the "one" and "two" categories were available. By defining three categories initially, and then using pairs of adjacent categories later, it was anticipated that criterion difficulties would be minimized, and such was the case.

\section{Simultaneity Threshold Task}

A second technique for minimizing difficulty with the "two" criteria was to obtain thresholds for the simultaneous perception of the vertical and horizontal bars contained within the disk. These sessions were run under the same stimulus conditions used for two-flash thresholds except that different response categories were employed. If $S$ perceived the vertical bar (right eye) and the horizontal bar (left eye) as occurring simultaneously, he responded, "cross." If he saw one bar, following subsequently by the other, he responded, "successive." If he detected a movement of the bar from a vertical position to the horizontal position, he reported "flop" (or "flip"). Three simultaneity threshold sessions, one for each flash duration, were run with each of the six Ss. This task provided a measure of binocular fusion unaffected by the possible problem of setting a criterion for a "two" response in the two-flash threshold situation

\section{Light Adaptation}

At the beginning of each session, $S$ was seated in front of a transilluminated milk Lucite screen, $27 \mathrm{~cm}$ high $\times 42.5 \mathrm{~cm}$ wide, which subtended a visual angle of $56 \mathrm{deg}$ high $\times 80$ deg wide when S's eyes were positioned at a distance of $25 \mathrm{~cm}$ away from its center. The luminance of this screen was $2 \mathrm{ft}-\mathrm{L}$. Five minutes of adaptation were given, during which time instructions were read. Intermittent light adaptation to this screen was administered throughout the course of a session $(2 \mathrm{~min}$ of adaptation per $5 \mathrm{~min}$ of task performance) in order to maintain a relatively constant level of adaptation and to prevent $S$ from detecting features of the alley other than the stimuli in Fig. 1. These periods served the additional purpose of providing rest intervals.

\section{Practice Sessions}

Seven 1-h practice sessions preceded the experimental sessions. These sessions served to insure that Ss were proficient at the brightness matching and depth shift tasks. The experiment proper required an additional 271 -h sessions.

\section{Depth Shifts \\ RESULTS}

All depth shift data were presented in terms of seconds of arc of shift. This angular measure of the perceived position of the disk was obtained as follows: If the mean perceived position of the disk with an IDI of $0 \mathrm{msec}$ were, for example, $700 \mathrm{~cm}$ for an $\mathrm{S}$ whose interpupillary distance was $6.4 \mathrm{~cm}$, a triangle could be constructed whose altitude was $700 \mathrm{~cm}$ and whose base was $6.4 \mathrm{~cm}$. With this information, the apex angle, $\alpha_{1}$, can readily be calculated. Similarly, if the mean perceived position of the disk with an IDI of $60 \mathrm{msec}$ were $730 \mathrm{~cm}$, the apex angle, $\alpha_{2}$, of the triangle thus formed can also be calculated. The difference $\alpha_{1}-\alpha_{2}$ would constitute the depth shift of the disk in seconds of arc when the IDI was $60 \mathrm{msec}$

Figure $2 \mathrm{~A}$ indicates the effect of both IDI and flash duration on depth shifts expressed in seconds of arc for all eight Ss. All shifts are positive. ${ }^{3}$ This means that the disk appeared to be farther away from $S$ when the IDI was greater than zero. It can be seen that flash durations of 20 and $40 \mathrm{msec}$ produced much smaller depth shifts than did a flash duration of 5 msec. 4
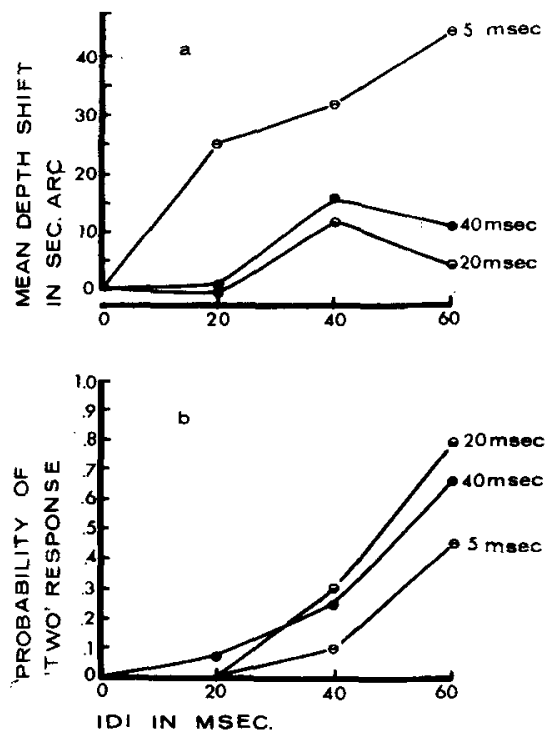

Fig. 2. (A) Mean depth shift as a function of interocular delay interval and disk duration $(\mathrm{N}=8)$. (B) Probability of a "two-flash" response as a function of interocular delay interval and disk duration $(\mathbf{N}=8)$.

\section{Two-Flash Thresholds}

In Fig. 2B, the probability of a two-flash response as a function of IDI and flash duration is plotted. These data were obtained concurrently with the depth shift data of Fig. 2A. The thresholds for the three durations from shortest to longest were $64.3,48.5$, and $52.0 \mathrm{msec}$, respectively. Since, for the 5 -msec flash duration, the probability of a two response never quite reached .5 , the value of $64.3 \mathrm{msec}$ was obtained by extrapolating the function. A comparison of the two parts of Fig. 2 makes evident the fact that large depth shifts were associated with large two-flash thresholds.

Figure 3 shows the mean two-flash thresholds, obtained by the method of constant stimuli, for six additional Ss. The line labeled 1-2 represents the mean threshold when $S$ was asked to discriminate between one vs two clearly separated flashes. The other line is for the case in which Ss were asked to discriminate between one flash and a "flick." Both criteria resulted in TFTs that decreased similarly as a function of flash duration. The "one-flick" criterion resulted in thresholds that were about $18 \mathrm{msec}$ lower than those for the "one-two" criterion. The general form of these functions, as well as the absolute magnitude of the thresholds, is quite similar to those indicated in Fig. 2B for the Ss who performed the depth shift task as well. 
Fig. 3. Mean two-flash threshold as a function of disk duration and response cirterion ( $N=6$, see text).

Fig. 4. Mean simultaneity thresh. Id as a function of disk duration and response criterion $(N=6$, see text).

\section{Simultaneity Thresholds}

Figure 4 presents the data from the simultaneity threshold sessions for the three flash durations and for the three respo. ise categories. The line labeled F-S represents the threshold for a shift from the "flop" to the "successive" response category. This criterion was not affected by flash duration. The line labeled C-S represents the threshold for a shift from the "cross" to the "successive" category, while the last line represents the shift from the "cross" to the "flop" category. Both of these criteria were affected by flash duration. Thresholds decreased with increasing flash duration in a manner similar to that of the two-flash thresholds of Fig. 2B.

It should be noted that while the stimulus conditions were appropriate for eliciting binocular rivalry under continuous viewing conditions, no reports of rivalry were ever obtained at short delay intervals where they might be expected to occur. This finding is consistent with that reported by Kaufman (1963). He found no binocular rivalry with stimulus exposures of $50 \mathrm{msec}$ in duration.

A comparison of the simultaneity and two-flash thresholds results with those of the depth-shift functions in Fig. 2A strongly supports the inference that large depth shifts are associated with durations that produce large simultaneity and two-flash thresholds. An examination of the individual data supports this inference. Ss who showed larger depth shifts tended to have larger ST and TFT thresholds. Kendall rank-order correlations were performed on the data of the eight original Ss for each flash duration-IDI combination. A positive correlation between the STs and depth-shift magnitudes of the Ss would support that inference made from the group data. Six of the nine correlations were positive, although only one reached statistical significance $(+.81, p=.015)$ for the $20 \mathrm{msec}$.

\section{DISCUSSION}

The results of this study do not support the hypothesis that depth shifts resulting from an interocular delay in stimulation are the result of a breakdown of binocular fusion, which renders the IDI test object (the disk in the present study) functionally monocular. The alternative hypothesis that depth shifts can result from an IDI within the temporal limits of binocular fusion is
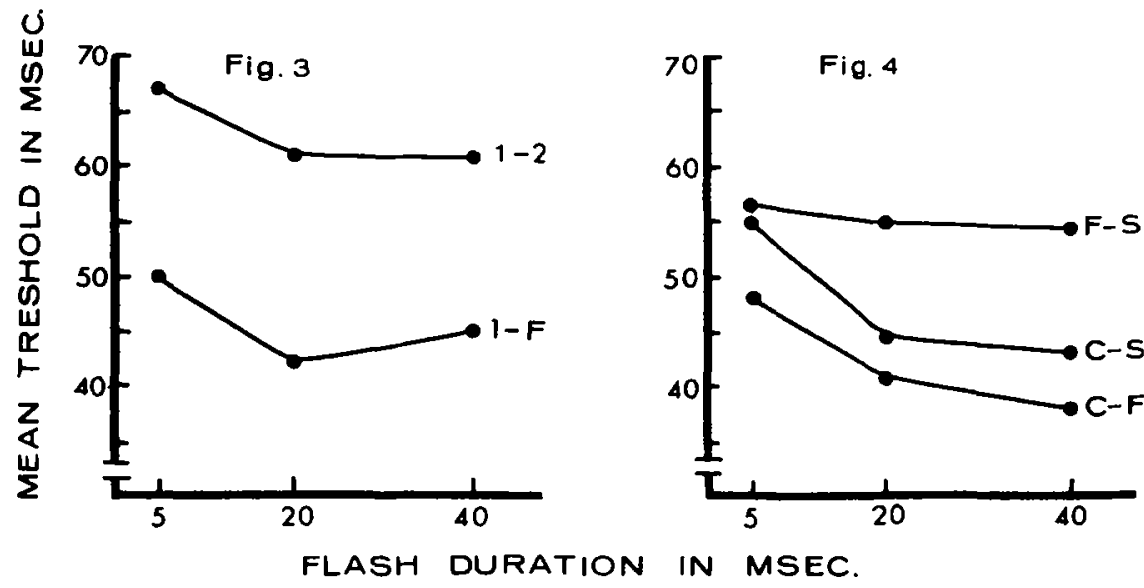

supported. A flash duration of $5 \mathrm{msec}$ that produced the largest two-flash thresholds also produced the largest depth shifts. This suggests that the greater the temporal gap over which the fusion can be maintained, the greater the possible magnitude of depth shifts.

If this conclusion is valid, the hypothesis in the introduction, that temporal disparity of stimulation has an effect on perceived depth equivalent to that of retinal (spatial) disparity within the temporal limits of binocular fusion, may be valid. An equivalence between retinal and temporal disparity suggests the possibility that spatial inputs are encoded, at least in part, in terms of a temporal dimension. If the coding of stereoscopic depth were entirely spatial, it would be expected that temporal disparities in stimulation, such as in the present study, would produce highly ambiguous perceptions of depth or a sharp discontinuity in the depth-shift function rather than the progressive shift in depth found in this study (see Fig. 2A) and in previous studies (Wist \& Gogel, 1966; Wist, 1968).

Some precedence exists for the concept of a temporal code mediating stereoscopic depth. Hering (1864) hypothesized that latency differences between the nasal and temporal hemiretinas mediated the perception of which of two points, $A$ and $B$, separated in depth is the nearer. Retinal disparity by itself specifies only that a depth interval exists. The same retinal disparity could be produced by $\mathbf{A}$ and $\mathbf{B}$ if $B$ were the nearer point. Bower (1966) reported that an interocular delay of only $1.5 \mathrm{msec}$ was sufficient to change perception from $A$ in front of $B$ to $B$ in front of $\mathbf{A}$. Unfortunately, a replication, to be reported later, of his study in our laboratory failed to produce the effect he reported.

It should be noted that temporal disparity is involved in the Pulfrich phenomenon (Pulfrich, 1922; Lit, 1949). In this case, however, it is clear that the temporal disparity produced by a luminance difference between the eyes, which results in a retinal latency difference, has its effect directly on retinal disparity. "Images" of the moving pendulum that are binocularly fused are more disparate because of the delay in perception in the eye with the reduced luminance. No effect occurs with a stationary pendulum, since no additional retinal disparity is produced by a delay under this condition. In the present study, no stimulus movement occurred, and furthermore, the interocular delay was selective: A temporal disparity existed only for the disk (see Fig. 1A); all other objects were viewed without delay. In the Pulfrich situation, all inputs in the filtered eye are delayed with respect to inputs in the normal eye.

Before an equivalence relation between temporal and retinal disparity could be regarded as established, other consequences of this hypothesis need to be tested. For example, it should be the case that the depth-shift threshold is smaller than the two-flash threshold. 5 Also, in order to describe this relation as one involving central mechanisms, the possibility of eye movements as a mediator for the depth shift effect must be ruled out. One study has been completed using IDIs of 0 and $64 \mathrm{msec}$. While the usual depth shift effect was obtained, fixation disparity measures did not differ for the 0 - and $64-\mathrm{msec}$ conditions, as would be expected if the eyes were moving with respect to each other during the IDI, thus modifying the retinal disparity of stimulation of the two retinas directly, by changing the proximal stimulus, rather than indirectly in terms of some central mechanism. A larger scale study in which fixation disparity will be measured for the entire range of IDIs that produce depth shifts must be finished 
before the possibility that eye movements are responsible for the depth shift effect can be entirely ruled out. One difficulty with eye movements as a mediator of the depth shift effect should be pointed out. The latency of response for eye movements is rather long compared to the delays at which reliable depth shifts occur. Westheimer and Mitchell (1956) reported eye-movement latencies of approximately $160 \mathrm{msec}$. With 5-msec flashes and a 60-msec IDI, the total elapsed time at the end of the second flash is only $70 \mathrm{msec}$. Thus, an eye movement induced by the first flash would not be initiated until 90 msec after stimulation was completed.

Still another possible account of the results must be ruled out before the equivalence hypothesis can be accepted. If one assumption is made, the data of this study can be regarded as supporting the binocular fusion breakdown hypothesis. In earlier studies (Wist \& Gogel, 1966; Wist, 1968), it was suggested that Panum's Case and the equidistance tendency interact to determine the direction of the depth shift. In the present study, if one assumes that the strength of the equidistance tendency increases with flash duration, the failure to obtain larger depth shifts for longer durations may reflect the increasing strength of the equidistance tendency between the test object and the standard. Although current evidence suggests that under reduced stimulus conditions the equidistance tendency grows over seconds and minutes rather than milliseconds (Lodge \& Wist, 1968; Wist \& Summons, 1969), this possibility cannot be entirely ruled out until the strength of the ET for the flash durations employed in the present study has been determined.

A last possibility must be considered. Although the results fail to support directly the hypothesis that depth shifts result from a breakdown of binocular fusion, they are not necessarily incompatible with it. The possibility of a "two-factor" account of the depth shift phenomenon must be entertained. On this account, one mechanism operates to produce depth shifts when IDIs are within the temporal limits of binocular fusion, and another operates at IDIs beyond these limits. Within the limits of fusion, such stimulus variables as the equidistance tendency, the relative potency of binocular and monocular cues, and relative size influence both depth shift magnitude and direction. Such a model would make sense of the data of an earlier study (Wist, 1968), in which it was found that the equidistance tendency, while strong enough to reverse the direction of shift in perceived depth location for a monocular test object, failed to produce a depth shift reversal for a binocular test object with an IDI of $64 \mathrm{msec}$. If the $64 \mathrm{msec}$ can be regarded as being within the limits of binocular fusion, as the data from the present study suggest, then it would be expected that only at IDI that were larger, say 96 or $128 \mathrm{msec}$, would a shift in direction as well as the magnitude of the depth shift take place. An experiment testing this prediction is currently in progress.

A final comment should be made concerning the bearing of the results on suppression theories of stereopsis (Verhoeff, 1935; Hochberg, 1964). According to these theories, binocular fusion of simultaneous inputs to the two eyes does not occur, but rather some portion of the input in one eye is suppressed at the time that a portion of the input in the corresponding position in the other retina is being seen. Thus, at the level of the visual system at which binocular integration occurs, there is available at any moment in time discharges from only one member of each pair of corresponding retinal points.

It is difficult to account for the data of this study by employing the concept of suppression. Consider the dichoptic two-flash threshold as a measure of binocular fusion. A suppression theory would regard this threshold as representing the temporal separation between inputs at which suppression of one of the two flashes no longer occurs. One flash is seen below this threshold because of the suppression of the other flash with short delays.

Since the suppression process would then be unaffected by delays smaller than the two-flash threshold, normal depth perception ought to occur for these delays. The results do not agree with this prediction. Depth shifts were found for these shorter delays. Furthermore, as noted earlier, no evidence of binocular rivalry was obtained in the present study during sessions in which simultaneity thresholds were obtained. Yet, if suppression were occurring, rivalry should certainly have been observed.

\section{REFERENCES}

BOWER, T. G. R. A local sign for depth. Nature, $1966,210,1081-1082$.

GOGEL, W. C. Equidistance tendency and its consequences. Psychological Bulletin, 1965, 64, 153-163.

HERING, E. Zur Lehre vom Ortsinne des Netzhaut. Leipzig: Engelmann, 1864.

HOCHBERG, J. Depth perception loss with local monocular suppression: $A$ problem in the explanation of stereopsis. Science, 1964,145 , 1334-1335.

IRELAND, F. H. An experimental investigation of the monocular and binocular two pulse interval. Unpublished doctoral dissertation, Fordham University, 1954.

KAUFMAN, L. On the spread of suppression and binocular rivalry. Vision Research, 1963, 3, 401-415.

LIT, A. The magnitude of the Pulfrich stereophenomenon as a function of binocular differences in intensity at various levels of illumination. American Journal of Psychology, $1949,62,159-181$.

LODGE, H., \& WIST, E. R. The growth of the equidistance tendency over time. Perception \& Psychophysics, 1968, 3, 97-103.

MAHNEKE, A. Foveal discrimination measured with two successive light flashes. Acta Ophthalmologia, 1958, 36, 3-11.

OGLE, K. N. The optical space sense. In $H$. Dawson (Ed.), Visual optics and the optical space sense. Vol. 4. New York: Academic Press, 1962. Pp. 374-376.

PULFRICH, C. Die Stereoskopie im Dientse der isochromen and heterochromen Photometrie. II. Anwendungen der neuen Methode. Naturwissenschaften, 1922, 33, 714-722.

ROUSH, R., \& HAMBURGER, F., JR. Light-flash generator. Electronics, 1948, 21, 100-102.

VERHOEFF, F. H. A new theory of binocular vision. Archives of Ophthalmology, 1935, 13 . 151-175.

WESTHEIMER, G., \& MITCHELL, A. M. Eye movement responses to convergence stimuli. A.M.A. Archives of Ophthalmology, 1956, 55, 848-856.

WIST, E. R. The influence of the equidistance tendency on depth shifts resulting from an inter-ocular delay in stimulation. Perception \& Psychophysics, 1968, 3, 89-92.

WIST, E. R., \& GOGEL, W. C. The effect of interocular delay and repetition interval on depth perception. Vision Research, 1966, 6, 325-334.

WIST, E. R., \& SUMMONS, E. The effect of relative size on the equidistance tendency. Paper read at the meetings of The Eastern Psychological Association, April 1969.

\section{NOTES}

1. This study was supported by Research Grant MH10361-01 from the National Institute of Mental Health and by Grant NB05528-02 from the National Institute of Neurological Diseases and Blindness of the U.S. Public Health Service. The data collection of Donald $H$. Mershon and Howard Lodge is acknowledged as well as the performance of the data analyses by Walter Martin of the Franklin \& Marshall Computer Center. A portion of the data of this study was reported at the Psychonomic Society meetings, St. Louis, October 1966.

2. Address: Franklin and Marshall College, Lancaster, Pennsylvania 17604

3. Only the data from the depth shifts sessions in which the IDIs were $0,20,40$, and $60 \mathrm{msec}$ will be reported here. The data for the $0-30$, 50-, and 70-msec sessions were in all important respects identical to those of the former sessions.

4. An analysis of variance indicated that the main effects of duration and IDI and the duration-IDI interaction were statistically significant $(\mathrm{p}<.01)$. An analysis of variance on the 20 - and $40-\mathrm{msec}$ flash durations resulted in nonsignificant $\mathrm{Fs}$.

5. A study to be reported at a later date supports this prediction.

(Accepted for publication October 8, 1969.) 